\title{
Lacosamide and Levetiracetam Have No Effect on Sharp-Wave Ripple Rate
}

\begin{abstract}
Jan Kudlacek ${ }^{1,2}$, Jan Chvojka ${ }^{1,2}$, Antonin Posusta', Lubica Kovacova ${ }^{1}$, Seung Bong Hong ${ }^{3,4}$, Shennan Weiss ${ }^{5}$, Kamila Volna ${ }^{6}$, Petr Marusic ${ }^{6}$, Jakub Otahal ${ }^{1 \dagger}$ and Premysl Jiruska ${ }^{1 \times+}$

'Department of Developmental Epileptology, Institute of Physiology, The Czech Academy of Sciences, Prague, Czechia, 2Department of Circuit Theory, Faculty of Electrical Engineering, Czech Technical University in Prague, Prague, Czechia, ${ }^{3}$ Department of Neurology, Samsung Medical Center, Samsung Advanced Institute for Health Sciences \& Technology (SAIHST), Sungkyunkwan University School of Medicine, Seoul, South Korea, ${ }^{4}$ Samsung Biomedical Research Institute, Seoul, South Korea, ${ }^{5}$ Department of Neurology, Thomas Jefferson University, Philadelphia, PA, United States, ${ }^{6}$ Department of Neurology, 2nd Faculty of Medicine, Charles University and Motol University Hospital, Prague, Czechia
\end{abstract}

Pathological high-frequency oscillations are a novel marker used to improve the delineation of epileptogenic tissue and, hence, the outcome of epilepsy surgery. Their practical clinical utilization is curtailed by the inability to discriminate them from physiological oscillations due to frequency overlap. Although it is well documented that pathological HFOs are suppressed by antiepileptic drugs (AEDs), the effect of AEDs on normal HFOs is not well known. In this experimental study, we have explored whether physiological HFOs (sharp-wave ripples) of hippocampal origin respond to AED treatment. The results show that application of a single dose of levetiracetam or lacosamide does not reduce the rate of sharp-wave ripples. In addition, it seems that these new generation drugs do not negatively affect the cellular and network mechanisms involved in sharp-wave ripple generation, which may provide a plausible explanation for the absence of significant negative effects on cognitive functions of these drugs, particularly on memory.

Keywords: high-frequency oscillations, sharp-wave ripples, levetiracetam, lacosamide, antiepileptic drugs, hippocampus, ripples, in vivo

\section{HIGHLIGHTS}

- Pathological high-frequency oscillations (pHFOs) represent electrographic biomarker of epileptogenic tissue.

- Current approaches are not able to distinguish pathological HFOs from physiological ones in intracranial recordings.

- Antiepileptic drugs decrease the rate of pHFOs.

- Levetiracetam or lacosamide do not decrease the rate of sharp-wave ripples-a representative of physiological HFOs.

- Pharmacological testing could be used to discriminate pathological and physiological HFOs.

\section{INTRODUCTION}

Pathological high-frequency oscillations (pHFOs) represent a new electrographic marker of epileptogenic tissue. Early after their discovery, pHFO analysis was introduced into the presurgical evaluation to better delineate the resection margin and to improve the outcome of surgery. pHFOs are classified according to their frequency into two main groups-ripples $(80-250 \mathrm{~Hz})$ and fast ripples $(250-600 \mathrm{~Hz})(1,2)$. Although fast ripples are considered more specific for epileptogenic tissue than ripples, both types of $\mathrm{pHFO}$ can localize the epileptogenic zone or seizure onset areas in humans 
who undergo exploration with invasive electrodes $(1,2)$. Several studies have demonstrated the beneficial effect of complete resection of the pHFO generating areas on surgical outcome (3-5). The practical utilization of pHFOs in presurgical evaluation is substantially hindered by the inability to differentiate them from physiological high-frequency oscillations, such as hippocampal sharp-wave ripples (SWRs) as they display substantial frequency overlap $(2,6,7)$. Currently, we do not have any effective tools, which can reliably discriminate between them. Identification of an approach to reliably discriminate between normal and pathological HFOs is a complex, but essential, issue to address if the properties of $\mathrm{pHFO}$ are to be fully utilized in clinical practice.

One of the features of pHFOs is their responsiveness to antiepileptic drugs (AEDs). In the chronic pilocarpine model of temporal lobe epilepsy, pHFOs and seizure rate decrease after treatment with levetiracetam (8) or lacosamide (9). In humans, withdrawal of AEDs is associated with increased rate of pHFOs (10). A pharmacological test using AEDs could be a plausible strategy to discriminate physiological oscillations from pathological ones, providing rate of physiological HFOs is not decreased by AEDs.

In this proof-of-principle study, we explored the impact of a single dose of lacosamide or levetiracetam on the rate of SWRs-a hippocampal representative of physiological HFOs (11). SWRs play a crucial role in the process of coordinated memory reactivation and formation of long-term memory (12). We have tested the hypothesis that the application of a single therapeutic dose of levetiracetam or lacosamide does not affect SWR rate.

\section{MATERIALS AND METHODS}

\section{Electrode Implantation and EEG Recording}

All experiments were performed under the Animal Care and Animal Protection Law of the Czech Republic fully compatible with the guidelines of the European Union directive 2010/63/EU. The protocol was approved by the Ethics Committee of The Czech Academy of Sciences (Project License No. 71/2016). Animals were housed in groups under standard conditions in a room with controlled temperature $\left(22 \pm 1^{\circ} \mathrm{C}\right)$ and $12 / 12 \mathrm{~h} \mathrm{light/dark}$ cycle. Eleven adult male Wistar rats weighing between 350 and $430 \mathrm{~g}$ were used in this study. The surgical preparation was performed under isoflurane anesthesia. The animals were implanted with bipolar twisted silver electrodes (120 $\mu \mathrm{m}$ in diameter, AM Systems, Inc., USA) bilaterally in the stratum pyramidale (AP: $-4.1, \mathrm{~L}: 2.2, \mathrm{D}: 2.5)$ and stratum radiatum (AP: $-4.6, \mathrm{~L}: 2.6, \mathrm{D}: 2.6)$ of the dorsal CA1 according to the stereotaxic atlas (13). The two contacts of each electrode were $0.5 \mathrm{~mm}$ apart. Two ground/reference jeweler's screws were placed over the cerebellum. Following a 5-day recovery period, animals were individually video-EEG monitored for 4 weeks continuously. Spontaneous electrographic activity was amplified, band-pass filtered $(0.1 \mathrm{~Hz}-1.6 \mathrm{kHz})$, and digitized at $5 \mathrm{kHz}$ using a RHD2132 32-channel amplifier chip (Intan Technologies, USA). After the end of the experiment, animals were humanely euthanized by an overdose of urethane, brains extracted, and processed to verify the positions of electrodes (Figure 1E).

\section{AED Treatment}

Each animal received sequential intraperitoneal injections of levetiracetam (Keppra ${ }^{\circledR}$, UCB, S.A., Brussels, Belgium), saline (control solution) of equal volume, lacosamide (Vimpat ${ }^{\circledR}$, UCB, S.A., Brussels, Belgium), and saline of equal volume. Additionally, three of the animals received diazepam (Apaurin ${ }^{\circledR}$, Krka, d. d., Novo Mesto, Slovenia) and saline of equal volume.

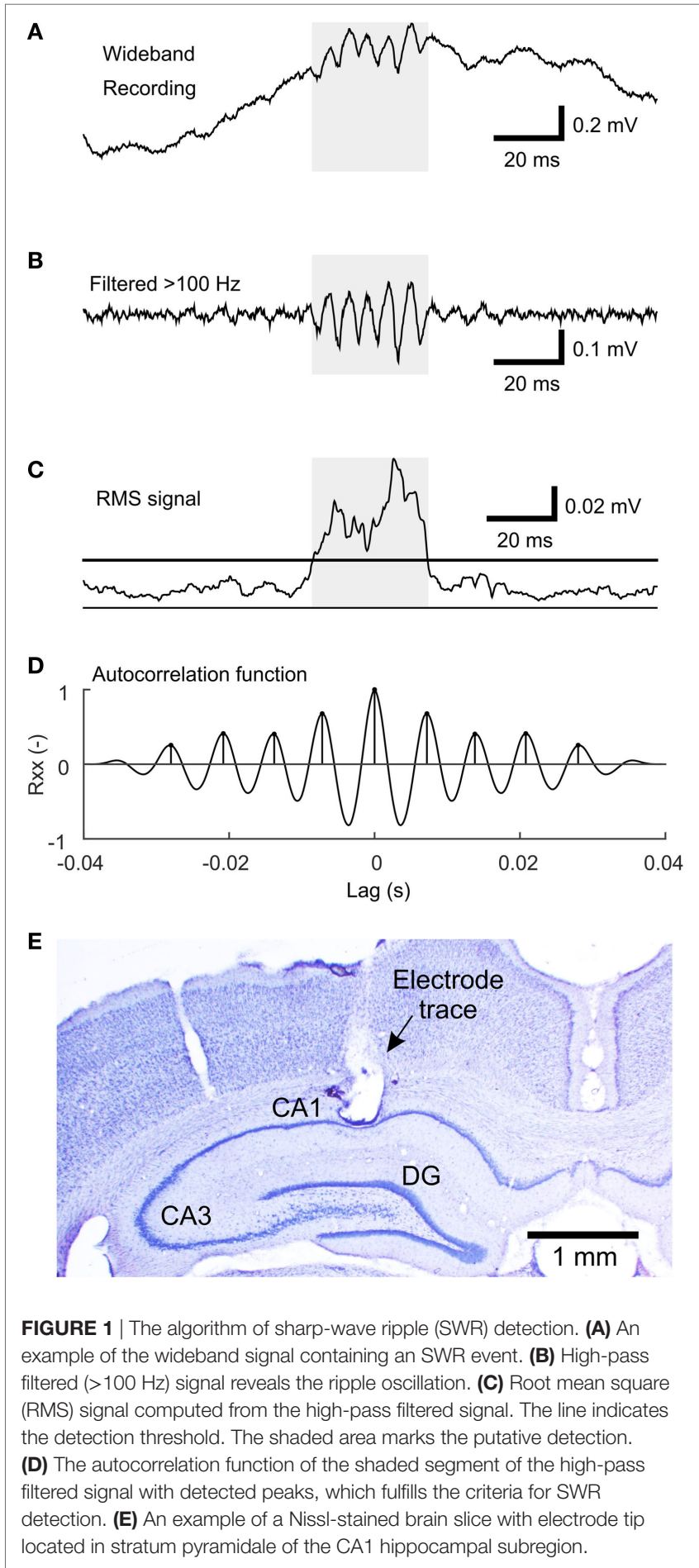


Diazepam acted as a positive control since it was shown to decrease the SWR rate in vivo (14). The interval after each injection, whether it was an AED or saline, was 3 days to allow maximal elimination of the drug from the body based on the known pharmacokinetics (14-16). The doses for levetiracetam and lacosamide were 80 and $30 \mathrm{mg} / \mathrm{kg}$, respectively. At comparable doses, these drugs were shown to effectively suppress pHFOs $(8,9)$. The dose of diazepam was $1 \mathrm{mg} / \mathrm{kg}$ which was shown to reduce or even suppress the SWR rate $(14,17)$. The sequence of injections was randomized between animals. In a given rat, every injection was administered at the same time of the day (10:30 a.m. or 2:00 p.m.).

\section{EEG Analysis}

EEGs were analyzed using custom made scripts in Matlab 2015a computing environment (Mathworks Inc., Natick, MA, USA). Two animals were removed from the analysis due to extremely noisy EEG signals. In one animal, the experiment was terminated prematurely due to the loss of the head cap prior to lacosamide injection. Therefore, the total number of animals used in the evaluation of the three drugs was nine for levetiracetam, eight for lacosamide, and three for diazepam. In each animal, SWRs were analyzed only in the channel in which SWRs displayed the highest amplitude. Correct positions of these electrodes in the hippocampal CA1 were verified histologically. We analyzed two epochs each lasting $2 \mathrm{~h}$. The first epoch was from 0.5 to $2.5 \mathrm{~h}$ after the injection and the second one was from 4.5 to $6.5 \mathrm{~h}$ after the injection. To determine the SWR rate during the same brain state, we extracted episodes of slow-wave sleep only, from each epoch, based on the presence of slow waves in the EEG and verified behaviorally in video recordings.

\section{SWR Detection}

Sharp-wave ripples were detected using the modified root mean square (RMS)-based approach (18). The signal was band-pass filtered (passband 100-200 Hz) using a FIR filter with a $40-\mathrm{Hz}$ wide transition band and stopband attenuation at $80 \mathrm{~dB}$. RMS value was calculated in $4 \mathrm{~ms}$ sliding windows. Only segments of at least $18 \mathrm{~ms}$ in duration with an RMS value $>1.5 \mathrm{SD}$ above the RMS mean were selected as putative SWRs. Events closer than $10 \mathrm{~ms}$ were treated as a single event. The next step of the detection procedure included estimation of the autocorrelation function in high-pass filtered segments $(>100 \mathrm{~Hz}$, otherwise the same parameters). Detections with at least seven peaks in the autocorrelation function with a lag corresponding to the SWR frequency band $(100-200 \mathrm{~Hz})$ and with the second peak of at least $25 \%$ of magnitude were selected. The main steps of the detection procedure are visualized in Figures 1A-D.

To evaluate the detector's performance, 24 randomly selected epochs of slow-wave sleep were labeled by an expert. Automatic detections were compared to the expert's labels. A true positive (TP) detection was defined as the one overlapping with an expert's label by at least $50 \%$ of the detection's duration. A false positive (FP) detection was a detection not fulfilling the criteria for TP detection. The number of false negatives (FN) was defined as the number of expert's labels, which had no overlap with any of the TP detections. Finally, sensitivity and positive predictive value (PPV) of the detector was calculated using the following equations:

$$
\begin{gathered}
\text { sensitivity }=\frac{\mathrm{TP}}{\mathrm{TP}+\mathrm{FN}} \\
\mathrm{PPV}=\frac{\mathrm{TP}}{\mathrm{TP}+\mathrm{FP}} .
\end{gathered}
$$

Sensitivity and PPV of the detector was 57 and 80\%, respectively. Detector's settings were optimized to achieve mainly high PPV to detect only true SWRs, omit ambiguous SWRs, and to minimize the risk of FP detections.

\section{Statistical Evaluation}

In this study, the primary hypothesis tested was that injections of selected AEDs do not affect SWR rates. This is in contrast to the vast majority of drug studies, which examine and test for the presence of an effect of the drug on specific phenomena, including HFOs. Thus, we had to implement an appropriate statistical method that tests equality and not an effect. In this study, we adopted the method recommended by Piaggio et al. (19). For each rat, each injection and each time after the injection we calculated average SWR rate during the slow-wave sleep epochs. Then, for each rat, each drug and each time after the injection, we calculated the ratio of SWR rate after AED injection to SWR rate after the corresponding saline injection. Since the data did not display normal distribution, we used a non-parametric approach. For each drug and each time after the injection, median of the ratios and its non-parametric confidence interval was calculated. The non-parametric confidence interval for the median is obtained as the $k$-th lowest and the $k$-th highest value from the sample. $k$ is determined so that the true population median lies within that interval with confidence equal or higher than required. In our study, we required at least $90 \%$ confidence. For sample sizes nine and eight we took $k=2$ which gives confidences of 96 and 93\%, respectively. For the three diazepam animals, we took the first and the last value as the confidence interval (widest possible), which gives up to $75 \%$ confidence (20). We obtained confidence intervals for all three drugs and two time windows after the injections. These six confidence intervals were compared to equality margins, which were set to 0.75 and 1.25 . If the confidence interval did not cross the equality margins, the AED was considered to have no effect on SWR rate.

Following equality testing, we performed statistical analysis to evaluate the possible presence of a statistically significant effect of AEDs on the SWR rate. In this step, we used the Wilcoxon signed-rank test on each set of the ratios. Before applying the test, the ratios were transformed by subtracting 1 so that a decrease in the SWR rate resulted in a negative number and vice versa. In the case of a non-significant result, post hoc power of the Wilcoxon signed-rank test was determined using SD of the data and location shift equal to the equality margin, i.e., $0.25(21,22)$.

\section{RESULTS}

In total 43,011 SWRs were detected with 4,779 $\pm 3,125$ events per animal. The average SWR rate was 16.0 events/min, which 
is congruent with studies focused on SWR in vivo (11, 23-25). SWR rates of all animals, after each injection, in both analyzed time windows, are shown in Figure 2. The data revealed the presence of individual variability in the SWR rate and response to the tested drugs. The crucial parameter for examination of the effect of an AED is the ratio between the SWR rate after AED injection and the SWR rate after control saline injection (Figure 3). The median ratio of the SWR rate between levetiracetam and

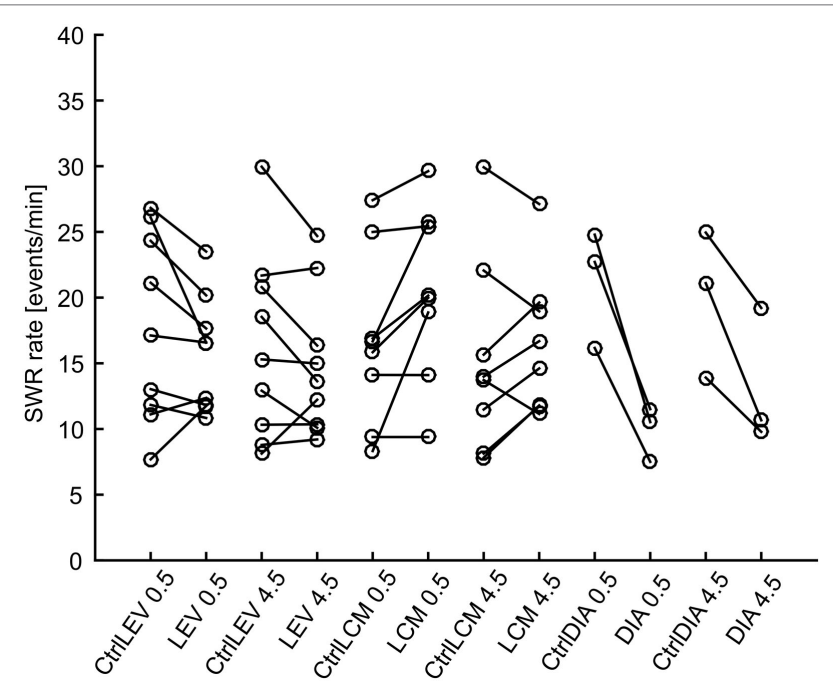

FIGURE 2 | Sharp-wave ripple (SWR) rates after various treatments. Lines connect data points from individual animals. LEV, levetiracetam; LCM, lacosamide; DIA, diazepam; CtrIXXX, injection of equivalent volume of saline; 0.5 , half an hour after injection, 4.5 , four and half hours after injection.

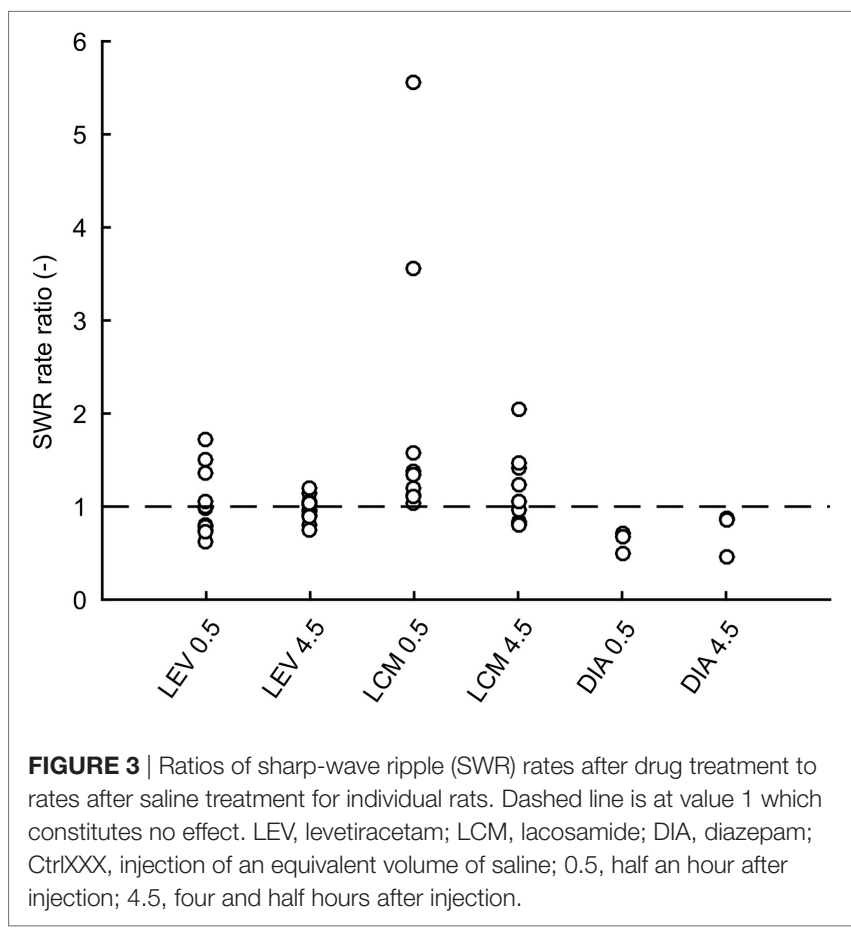

the control was 0.91 and 0.98 for $30 \mathrm{~min}$ and $4.5 \mathrm{~h}$ after the injection, respectively ( $n=9$ animals). For levetiracetam, the ratios' confidence intervals did not cross the equality margins $30 \mathrm{~min}$ and $4.5 \mathrm{~h}$ after injection (Figure 4). Therefore, SWR rates after levetiracetam and saline treatment can be considered equal within the equality margins. The Wilcoxon signed-rank test for ratios was non-significant for $30 \mathrm{~min}(p=0.30)$ and $4.5 \mathrm{~h}$ after the injection $(p=0.57)$, with a post hoc power of $88 \%$. For lacosamide, the median ratio of the SWR rate was 1.14 and 1.22 for each time epoch ( $n=8$ animals). The confidence intervals of the SWR rate ratios crossed the equality margins in both time windows. The Wilcoxon signed-rank test demonstrated a significant increase in SWR rate ratio $30 \mathrm{~min}$ after injection of lacosamide $(p=0.039)$. After $4.5 \mathrm{~h}$ the effect of lacosamide was non-significant (Wilcoxon signed-rank test; $p=0.11$; power $=85 \%)$. Diazepam, which was used as a positive control, reduced the SWR rate compared to the equivalent volume of saline in all rats by $>50 \%$ with a median ratio of 0.46 and 0.70 for each time window (Figure 4). However, statistical significance could not be reached due to the small number of rats ( $n=3$ animals; Wilcoxon signed-rank test; $p=0.25$; power $=46 \%)$.

\section{DISCUSSION}

We have shown that a single dose of levetiracetam and lacosamide does not reduce the rate of SWRs-a representative of physiological HFOs. The ability to reliably differentiate between pathological and physiological HFOs represents a crucial step

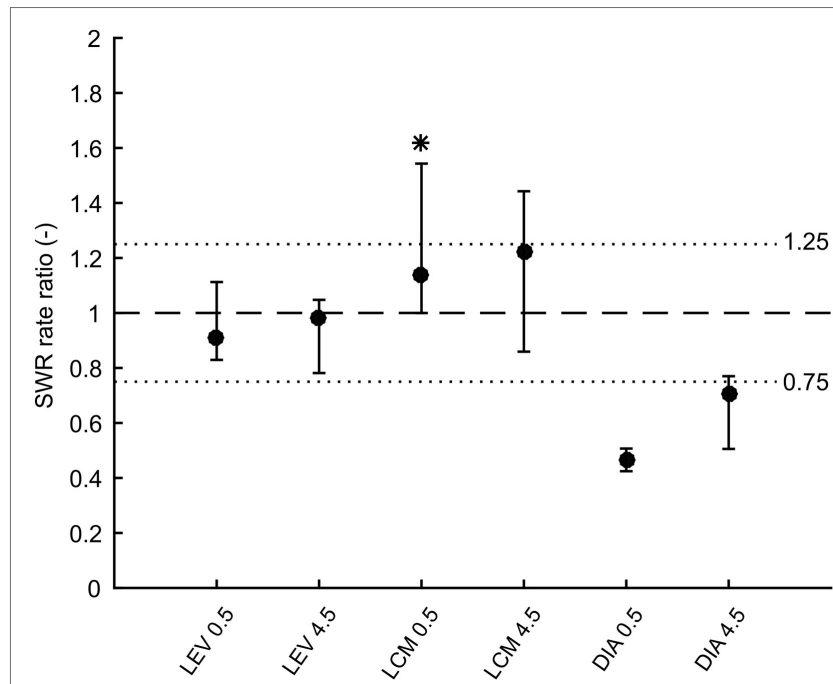

FIGURE 4 | Medians of ratios of sharp-wave ripple (SWR) rates after drug injection to rates after corresponding saline injection. Error bars represent non-parametric confidence intervals of the medians. Dashed line is at value 1 which constitutes no effect. Dotted lines at values 0.75 and 1.25 represent equality margins. LEV confidence intervals are at $96 \%$ confidence and do not cross the equality margins. LCM significantly increases SWR rate $0.5 \mathrm{~h}$ after administration, but $4.5 \mathrm{~h}$ after administration the effect dissipates. Confidence intervals are $93 \%$. DIA markedly reduces SWR rate $0.5 \mathrm{~h}$ after administration and $4.5 \mathrm{~h}$ after administration the effect slowly dissipates. Confidence intervals are $75 \%$. LEV, levetiracetam; LCM, lacosamide; DIA, diazepam. 
toward the clinical utilization of pHFOs as biomarkers of the epileptogenic zone $(2,7)$. Epileptic pHFOs display a spectral overlap with physiological HFOs such as SWRs or fast gamma activity. Fast ripples are considered to be exclusively of epileptic origin, but physiological activity with a frequency around $\sim 600 \mathrm{~Hz}$ has been described in the neocortex. Matsumoto and colleagues described features, which discriminated task-related physiological HFOs from pathological ones (26). Pathological HFOs were characterized by a higher spectral mean, longer duration, and lower mean frequency. Other studies tried to discriminate HFOs according to their spatial distribution, relationship with sleep phases (27), background activity (28), phase relationship with slow waves (29), or using cognitive tasks (30). However, the practical implementation of these criteria is challenging. Experimental treatment with levetiracetam or lacosamide has been shown to reduce the both ripples and fast ripples in the pilocarpine model of temporal lobe epilepsy, even though the effects were region specific $(8,9)$. In this in vivo study, we demonstrate that levetiracetam does not decrease the rate of hippocampal SWRs. The confidence intervals were set to tolerate a $25 \%$ change in SWR rate after the treatment. Studies which explored the effect of levetiracetam and lacosamide on pHFOs showed that these drugs reduced the pathological ripple rate in hippocampal structures by an average of $57 \%$ for levetiracetam and by $43 \%$ for lacosamide $(8,9)$. Although we studied the effect of these AEDs only on specific subtype of physiological HFOs, these experimental results suggest that the procedure of pharmacological testing can be a plausible approach to facilitate discrimination between physiological and pathological HFOs. Both drugs are used very frequently and their withdrawal and subsequent introduction is a very common procedure in patients undergoing invasive explorations. As an alternative, the introduction of AEDs could be replaced by an intravenous application of levetiracetam at the end of monitoring. HFOs that are not altered by AEDs can be considered to be physiological HFOs.

The absence of a major effect of the AEDs on the rate of SWRs and suppression of pHFOs can be explained by the currently known mechanisms of action of the drugs and cellular mechanisms of HFOs $(31,32)$. SWRs are oscillations that reflect the activation of large neuronal ensembles in the hippocampal CA1 region, particularly during sleep. On the cellular level, the oscillation depends on the precise interaction between pyramidal cells and inhibitory interneurons. The fast inhibitory postsynaptic potentials on the membrane of principal neurons play a key role in the coordination of principal cell firing (11). Pyramidal cells fire heterogeneously during the SWR; some cells can fire during every successive event, while other cells can fire only occasionally $(17,33)$. The sequence of neuronal activity during SWRs replays the sequence of neuronal activation during behavioral tasks, and it is considered to represent a mechanism of memory reactivation and long-term memory formation $(12,34)$. The cellular dynamics of pHFOs in both ripple and fast ripple bands differs and is more uniform $(31,32)$. During each $\mathrm{pHFO}$, a large population of cells generates a burst of high-frequency action potentials superimposed on a large depolarizing envelope (35-37). While high-frequency spiking depends mainly on fast sodium channel kinetics, the depolarizing envelope is associated with an increase in intracellular calcium via the opening of voltage-gated calcium channels or activation of non-NMDA and NMDA receptors. Lacosamide modifies voltage-gated sodium channel kinetics required for fast action potential firing, and it affects only neurons which are active or depolarized for prolonged periods of time and thus spares physiological functions (38). Therefore, pathological pHFOs are more susceptible to its effects than SWRs. The levetiracetam binds to SV2A, which is involved in trafficking and fusion of synaptic vesicles (39). It seems that levetiracetam reduces the vesicle release that is important for synaptic neurotransmission (40). It also partially blocks N-type calcium channels (41). However, the exact mechanisms of its actions are not well known, but we can assume that levetiracetam is capable of interfering with cellular processes involved in intense neuronal firing and pHFOs.

The absence of the effect of levetiracetam and lacosamide on the mechanisms of SWRs, in general, can also be deduced indirectly from the lack of their negative effect on cognitive functions including memory. It is well established that any drug or procedure, which has the capacity to interfere with the cellular mechanisms underlying the genesis of SWRs, also has the capacity to induce a memory deficit $(42,43)$. Levetiracetam is currently the drug of first choice, and lacosamide has also shown its therapeutic benefits as add-on therapy early after its introduction into epilepsy therapy. Both drugs are well tolerated by patients and demonstrate low adverse effects and an absence of a significant impact on cognition and memory (44-47). From electrophysiological perspective, these observations are supported by our experimental study, which demonstrates the absence of any suppressive effect of these drugs on the SWR generation. Moreover, lacosamide was shown to increase the speed of complex visual information processing (48), which may be in agreement with the transiently increased SWR rate observed in this study.

A possible limitation of this study is the single-dose scheme of drug administration. We might have missed the time period of SWR suppression by the drug. However, based on the known pharmacokinetics and mechanism of action of these drugs this seems unlikely $(15,16,38,49)$. Another possibility is that the drug was cleared from the body before its concentration in the brain could have reached sufficient intrathecal levels to influence the SWR rate. However, we used the same dose that was administered daily in studies exploring the effect of lacosamide and levetiracetam on pHFOs $(8,9)$ and pharmacokinetic studies showed that these drugs reach maximal brain concentration within $2 \mathrm{~h}$ after application $(15,16)$. Another weakness of the study is the single-dose application. We cannot exclude that prolonged or chronic application of these drugs may induce long-term changes, which would affect the properties of SWRs.

In conclusion, we have shown that levetiracetam does not change the rate of SWRs and lacosamide transiently increases it. Hence, these drugs can be considered for pharmacological testing to distinguish physiological versus pHFOs. Levetiracetam or lacosamide could be introduced toward the end of invasive exploration, and the response of HFOs to the drug introduction could be used to determine whether the HFOs are pathological or physiological. Moreover, our results are congruent with studies showing no negative effect of these drugs on cognition. 


\section{ETHICS STATEMENT}

This study was carried out in accordance with the recommendations of the Animal Care and Animal Protection Law of the Czech Republic fully compatible with the guidelines of the European Union directive 2010/63/EU. The protocol was approved by the Ethics Committee of The Czech Academy of Sciences (Project License No. 71/2016).

\section{AUTHOR CONTRIBUTIONS}

Conceived and designed the experiments: PJ, JO, PM, and SH. Performed the experiments: JC, JK, and LK. Analyzed the data:

\section{REFERENCES}

1. Zijlmans M, Jiruska P, Zelmann R, Leijten FS, Jefferys JG, Gotman J. Highfrequency oscillations as a new biomarker in epilepsy. Ann Neurol (2012) 71:169-78. doi:10.1002/ana.22548

2. Frauscher B, Bartolomei F, Kobayashi K, Cimbalnik J, van 't Klooster MA, Rampp S, et al. High-frequency oscillations: the state of clinical research. Epilepsia (2017) 58:1316-29. doi:10.1111/epi.13829.

3. Jacobs J, Zijlmans M, Zelmann R, Chatillon CE, Hall J, Olivier A, et al. Highfrequency electroencephalographic oscillations correlate with outcome of epilepsy surgery. Ann Neurol (2010) 67:209-20. doi:10.1002/ana.21847

4. Cho JR, Koo DL, Joo EY, Seo DW, Hong SC, Jiruska P, et al. Resection of individually identified high-rate high-frequency oscillations region is associated with favorable outcome in neocortical epilepsy. Epilepsia (2014) 55:1872-83. doi:10.1111/epi.12808

5. Holler Y, Kutil R, Klaffenbock L, Thomschewski A, Holler PM, Bathke AC, et al. High-frequency oscillations in epilepsy and surgical outcome. A metaanalysis. Front Hum Neurosci (2015) 9:574. doi:10.3389/fnhum.2015. 00574

6. Engel J Jr, Bragin A, Staba R, Mody I. High-frequency oscillations: what is normal and what is not? Epilepsia (2009) 50:598-604. doi:10.1111/j. 1528-1167.2008.01917.x

7. Cimbalnik J, Kucewicz MT, Worrell G. Interictal high-frequency oscillations in focal human epilepsy. Curr Opin Neurol (2016) 29:175-81. doi:10.1097/ WCO.0000000000000302

8. Levesque M, Behr C, Avoli M. The anti-ictogenic effects of levetiracetam are mirrored by interictal spiking and high-frequency oscillation changes in a model of temporal lobe epilepsy. Seizure (2015) 25:18-25. doi:10.1016/j. seizure.2014.11.008

9. Behr C, Levesque M, Ragsdale D, Avoli M. Lacosamide modulates interictal spiking and high-frequency oscillations in a model of mesial temporal lobe epilepsy. Epilepsy Res (2015) 115:8-16. doi:10.1016/j.eplepsyres.2015. 05.006

10. Zijlmans M, Jacobs J, Zelmann R, Dubeau F, Gotman J. High-frequency oscillations mirror disease activity in patients with epilepsy. Neurology (2009) 72:979-86. doi:10.1212/01.wnl.0000344402.20334.81

11. Ylinen A, Bragin A, Nadasdy Z, Jando G, Szabo I, Sik A, et al. Sharp wave-associated high-frequency oscillation $(200 \mathrm{~Hz})$ in the intact hippocampus: network and intracellular mechanisms. J Neurosci (1995) 15:30-46.

12. Buzsáki G. Hippocampal sharp wave-ripple: a cognitive biomarker for episodic memory and planning. Hippocampus (2015) 25:1073-188. doi:10.1002/ hipo. 22488

13. Paxinos G, Watson C. The rat brain. 4th ed. The Rat Brain in Stereotaxic Coordinates. San Diego, CA: Academic Press, Inc (1998). 474 p.

14. Ponomarenko AA, Korotkova TM, Sergeeva OA, Haas HL. Multiple GABAA receptor subtypes regulate hippocampal ripple oscillations. Eur J Neurosci (2004) 20:2141-8. doi:10.1111/j.1460-9568.2004.03685.x

15. Tong X, Patsalos PN. A microdialysis study of the novel antiepileptic drug levetiracetam: extracellular pharmacokinetics and effect on taurine in rat brain. Br J Pharmacol (2001) 133:867-74. doi:10.1038/sj.bjp.0704141
JC, JK, LK, AP, PJ, and JO. Wrote the paper: JC, JK, PJ, KV, SW, $\mathrm{PM}$, JO, and $\mathrm{SH}$.

\section{ACKNOWLEDGMENTS}

This study was supported by grants from the Czech Science Foundation GACR 14-02634S (to PJ), GACR 15-08565S (to JO), Neuron Fund for Support of Science 001/2012 (to PJ), the Ministry of Health of the Czech Republic AZV 15-29835A (to PM and PJ), 17-28427A (to PJ), and Korea Health Technology R\&D Project through the Korea Health Industry Development Institute (KHIDI), funded by the Ministry of Health \& Welfare, Republic of Korea (grant number: HI16C1643) (to SH).

16. Koo TS, Kim SJ, Ha DJ, Baek M, Moon H. Pharmacokinetics, brain distribution, and plasma protein binding of the antiepileptic drug lacosamide in rats. Arch Pharm Res (2011) 34:2059-64. doi:10.1007/s12272-011-1208-7

17. Buzsaki G, Horvath Z, Urioste R, Hetke J, Wise K. High-frequency network oscillation in the hippocampus. Science (1992) 256:1025-7. doi:10.1126/ science. 1589772

18. Staba RJ, Wilson CL, Bragin A, Fried I, Engel J Jr. Quantitative analysis of high-frequency oscillations $(80-500 \mathrm{~Hz})$ recorded in human epileptic hippocampus and entorhinal cortex. J Neurophysiol (2002) 88:1743-52. doi:10.1152/ jn.00322.2002

19. Piaggio G, Elbourne DR, Pocock SJ, Evans SJ, Altman DG, CONSORT Group. Reporting of noninferiority and equivalence randomized trials: extension of the CONSORT 2010 statement. JAMA (2012) 308:2594-604. doi:10.1001/ jama.2012.87802

20. Hogg RV, Tanis EA. Probability and Statistical Inference. New Jersey: Prentice Hall (2006)

21. Lehmann EL. Nonparametrics: Statistical Methods Based on Ranks. San Francisco: Holden-day, Inc. (1975).

22. Shieh G, Jan S-L, Randles RH. Power and sample size determinations for the Wilcoxon signed-rank test. J Stat Comput Simul (2007) 77:717-24. doi:10.1080/10629360600635245

23. Csicsvari J, Hirase H, Czurko A, Mamiya A, Buzsaki G. Fast network oscillations in the hippocampal CA1 region of the behaving rat. J Neurosci (1999) 19:Rc20.

24. Eschenko O, Ramadan W, Mölle M, Born J, Sara SJ. Sustained increase in hippocampal sharp-wave ripple activity during slow-wave sleep after learning. Learn Mem (2008) 15:222-8. doi:10.1101/lm.726008

25. Cheng S, Frank LM. New experiences enhance coordinated neural activity in thehippocampus.Neuron (2008) 57:303-13.doi:10.1016/j.neuron.2007.11.035

26. Matsumoto A, Brinkmann BH, Matthew Stead S, Matsumoto J, Kucewicz MT, Marsh WR, et al. Pathological and physiological high-frequency oscillations in focal human epilepsy. J Neurophysiol (2013) 110:1958-64. doi:10.1152/ jn.00341.2013

27. Frauscher B, von Ellenrieder N, Dubeau F, Gotman J. EEG desynchronization during phasic REM sleep suppresses interictal epileptic activity in humans. Epilepsia (2016) 57:879-88. doi:10.1111/epi.13389

28. Kerber K, Dumpelmann M, Schelter B, Le Van P, Korinthenberg R, Schulze-Bonhage A, et al. Differentiation of specific ripple patterns helps to identify epileptogenic areas for surgical procedures. Neurophysiol Clin (2014) 125:1339-45. doi:10.1016/j.clinph.2013.11.030

29. von Ellenrieder N, Frauscher B, Dubeau F, Gotman J. Interaction with slow waves during sleep improves discrimination of physiologic and pathologic high-frequency oscillations (80-500 Hz). Epilepsia (2016) 57:869-78. doi:10.1111/epi.13380

30. Axmacher N, Elger CE, Fell J. Ripples in the medial temporal lobe are relevant for human memory consolidation. Brain (2008) 131:1806-17. doi:10.1093/ brain/awn 103

31. Jefferys JG, Menendez de la PL, Wendling F, Bragin A, Avoli M, Timofeev I, et al. Mechanisms of physiological and epileptic HFO generation. Prog Neurobiol (2012) 98:250-64. doi:10.1016/j.pneurobio.2012.02.005 
32. Jiruska P, Alvarado-Rojas C, Schevon CA, Staba R, Stacey W, Wendling F, et al. Update on the mechanisms and roles of high-frequency oscillations in seizures and epileptic disorders. Epilepsia (2017) 58:1330-9. doi:10.1111/epi.13830

33. Csicsvari J, Hirase H, Czurko A, Mamiya A, Buzsaki G. Oscillatory coupling of hippocampal pyramidal cells and interneurons in the behaving rat. J Neurosci (1999) 19:274-87.

34. Csicsvari J, O'Neill J, Allen K, Senior T. Place-selective firing contributes to the reverse-order reactivation of CA1 pyramidal cells during sharp waves in open-field exploration. Eur J Neurosci (2007) 26:704-16. doi:10.1111/j. 1460-9568.2007.05684.x

35. Bragin A, Wilson CL, Engel J Jr. Chronic epileptogenesis requires development of a network of pathologically interconnected neuron clusters: a hypothesis. Epilepsia (2000) 41(Suppl 6):S144-52. doi:10.1111/j.1528-1157.2000. tb01573.x

36. Foffani G, Uzcategui YG, Gal B, Menendez de la PL. Reduced spike-timing reliability correlates with the emergence of fast ripples in the rat epileptic hippocampus. Neuron (2007) 55:930-41. doi:10.1016/j.neuron.2007.07.040

37. Ibarz JM, Foffani G, Cid E, Inostroza M, Menendez de la PL. Emergent dynamics of fast ripples in the epileptic hippocampus. JNeurosci (2010) 30:16249-61. doi:10.1523/JNEUROSCI.3357-10.2010

38. Rogawski MA, Tofighy A, White HS, Matagne A, Wolff C. Current understanding of the mechanism of action of the antiepileptic drug lacosamide. Epilepsy Res (2015) 110:189-205. doi:10.1016/j.eplepsyres.2014.11.021

39. Lynch BA, Lambeng N, Nocka K, Kensel-Hammes P, Bajjalieh SM, Matagne A, et al. The synaptic vesicle protein SV2A is the binding site for the antiepileptic drug levetiracetam. Proc Natl Acad Sci U S A (2004) 101:9861-6. doi:10.1073/ pnas.0308208101

40. Yang XF, Weisenfeld A, Rothman SM. Prolonged exposure to levetiracetam reveals a presynaptic effect on neurotransmission. Epilepsia (2007) 48:1861-9. doi:10.1111/j.1528-1167.2006.01132.x

41. Lukyanetz EA, Shkryl VM, Kostyuk PG. Selective blockade of N-type calcium channels by levetiracetam. Epilepsia (2002) 43:9-18. doi:10.1046/j. 1528-1157.2002.24501.x

42. Girardeau G, Benchenane K, Wiener SI, Buzsaki G, Zugaro MB. Selective suppression of hippocampal ripples impairs spatial memory. Nat Neurosci (2009) 12:1222-3. doi:10.1038/nn.2384
43. Ego-Stengel V, Wilson MA. Disruption of ripple-associated hippocampal activity during rest impairs spatial learning in the rat. Hippocampus (2010) 20:1-10. doi:10.1002/hipo.20707

44. Lopez-Gongora M, Martinez-Domeno A, Garcia C, Escartin A. Effect of levetiracetam on cognitive functions and quality of life: a one-year follow-up study. Epileptic Disord (2008) 10:297-305. doi:10.1684/epd.2008.0227

45. Javed A, Cohen B, Detyniecki K, Hirsch LJ, Legge A, Chen B, et al. Rates and predictors of patient-reported cognitive side effects of antiepileptic drugs: an extended follow-up. Seizure (2015) 29:34-40. doi:10.1016/j.seizure.2015.03.013

46. Lancman ME, Fertig EJ, Trobliger RW, Perrine K, Myers L, Iyengar SS, et al. The effects of lacosamide on cognition, quality-of-life measures, and quality of life in patients with refractory partial epilepsy. Epilepsy Behav (2016) 61:27-33. doi:10.1016/j.yebeh.2016.04.049

47. Schoenberg MR, Rum RS, Osborn KE, Werz MA. A randomized, double-blind, placebo-controlled crossover study of the effects of levetiracetam on cognition, mood, and balance in healthy older adults. Epilepsia (2017) 58:1566-74. doi:10.1111/epi.13849

48. IJff DM, van Veenendaal TM, Majoie HJ, de Louw AJ, Jansen JF, Aldenkamp AP. Cognitive effects of lacosamide as adjunctive therapy in refractory epilepsy. Acta Neurol Scand (2015) 131:347-54. doi:10.1111/ane.12372

49. Deshpande LS, Delorenzo RJ. Mechanisms of levetiracetam in the control of status epilepticus and epilepsy. Front Neurol (2014) 5:11. doi:10.3389/ fneur.2014.00011

Conflict of Interest Statement: PM has received honoraria from UCB as a speaker and advisory board member. The remaining authors have no conflicts of interest. We confirm that we have read the Journal's position on issues involved in ethical publication and affirm that this report is consistent with those guidelines.

Copyright (c) 2017 Kudlacek, Chvojka, Posusta, Kovacova, Hong, Weiss, Volna, Marusic, Otahal and Jiruska. This is an open-access article distributed under the terms of the Creative Commons Attribution License (CC BY). The use, distribution or reproduction in other forums is permitted, provided the original author(s) or licensor are credited and that the original publication in this journal is cited, in accordance with accepted academic practice. No use, distribution or reproduction is permitted which does not comply with these terms. 